\title{
ARTICULO DE INVESTIGACION \\ La fragmentación del bosque mesófilo de montaña y patrones de uso del suelo en la región oeste de Xalapa, Veracruz, México
}

\author{
Guadalupe Williams-Linera ${ }^{1}$ \\ Robert H. Manson ${ }^{1}$ \\ Eduardo Isunza Vera ${ }^{2}$
}

\begin{abstract}
RESUMEN
La destrucción del bosque de niebla en la región oeste de Xalapa, Veracruz, se ha acelerado considerablemente en las últimas décadas. El objetivo de este estudio fue determinar el grado y patrón de fragmentación del bosque en la región. A partir de fotointerpretación y digitalización de contornos en un fotomosaico de treinta y tres fotografías aéreas escala 1:20 000 de INEGI tomadas en 1993, se obtuvieron mapas de vegetación y uso del suelo. Se integró información adicional sobre pendiente, orientación de la pendiente y poblaciones en un sistema de información geográfica. En el área de estudio (12 843 ha), quedan 19 fragmentos de bosque no perturbado que cubren únicamente el $10 \%$ del área. Los usos del suelo dominantes fueron pastizal (37\%), urbano (18\%), vegetación secundaria (17\%) y bosque perturbado (17\%). La poca superficie plana en la región (3.2\%) está relativamente más utilizada para usos urbanos y pastizales. El bosque no perturbado se encuentra sobre pendientes muy fuertes, con orientación más al norte, rodeado por potreros, bosque perturbado y vegetación secundaria; el efecto de borde reduce el área real del bosque en un 15-54\% adicional, dependiendo del tamaño del fragmento. El $90 \%$ del bosque de niebla ya ha sido destruido y el resto está en peligro de desaparecer. Se requiere un plan de desarrollo regional que considere la conservación del bosque por su biodiversidad y como fuente de servicios ambientales, que proteja los fragmentos de bosque no perturbado, promueva la restauración ecológica de bosque perturbado y establezca corredores que conecten al bosque remanente.
\end{abstract}

PALABRAS CLAVE:

Bosque de niebla, efecto de borde, fragmentación del bosque, SIG, cambio de uso del suelo.

\begin{abstract}
The destruction of tropical montane cloud forest in the western region of Xalapa, Veracruz, has accelerated considerably in the last few decades. This paper objective was to determine the degree and pattern of fragmentation of cloud forest in this region. Thirty-three aerial photographs, scale 1: 20000 (INEGI, 1993), were digitized and photo-interpreted to generate maps of vegetation and land-use. Additional information on slope, slope aspect, and human settlements was incorporated in a geographical information system (GIS). In the study area (12 $843 \mathrm{ha}$ ), nineteen fragments of undisturbed forest remain, which occupy just $10 \%$ of the region. The dominant land uses are pasture $(37 \%)$, urban areas $(18 \%)$, secondary vegetation $(17 \%)$ and disturbed forest $(17 \%)$. The few flat areas available in the region (3.2\%) are occupied by urban zones and pastures, while undisturbed forest is found mostly on steep slopes, with a northern exposure, located far from human activity. Forest fragments are surrounded by pastures, disturbed forest and secondary vegetation which may produce strong edge effects and reduce the overall undisturbed forest cover by another $15-54 \%$, depending on the size of forest fragments. Results indicate that $90 \%$ of the forest in the region has already been destroyed and what remains is in danger of disappearing. A regional development plan is required which considers the importance of cloud forests as biodiversity reservoirs and providers of key environmental services, protects existing fragments of undisturbed forest, and simultaneously promotes the ecological restoration of disturbed forest as well as the establishment of corridors connecting forest remnants.
\end{abstract}

KEY WORDS:

Edge effect, forest fragmentation, GIS, land use change, tropical montane cloud forest. 


\section{INTRODUCCIÓN}

El bosque mesófilo de montaña mantiene una gran biodiversidad y es sumamente importante por los servicios ambientales que proporciona a los asentamientos humanos vecinos. Este bosque se encuentra aislado física y climatológicamente en la parte media de las montañas, usualmente dentro de un estrecho rango altitudinal de $300 \mathrm{~m}$, dependiendo de la altura en donde se forman las nubes (Aldrich et al., 2000). En México, la importante biodiversidad del bosque de niebla se debe principalmente a la combinación de humedad alta y temperaturas templadas que han creado un ambiente favorable para la coexistencia de la flora templada y la neotropical, así como para la evolución y mantenimiento de la diversidad de especies de plantas y animales, muchas de las cuales son exclusivas de este ecosistema (Rzedowski, 1993, 1996; Challenger, 1998).

El bosque mesófilo de montaña ocupa menos del $1 \%$ de la superficie total de México. Sin embargo, se estima que lo componen de 2500 a 3000 especies de plantas (Rzedowski, 1996), lo cual representa entre 10 y $12 \%$ de todas las especies de plantas que existen en México y hace de este tipo de bosque el más diverso en México por unidad de superficie. Además, el bosque mesófilo de montaña cuenta con el número más alto de especies de mamíferos que cualquier otro tipo de bosque (95 especies; Fa y Morales, 1993) y una alta tasa de endemismo de plantas $(30 \%$ de las especies), reptiles (39\%), anfibios (33\%), aves $(71 \%)$ y mamíferos (53\%; FloresVillela y Gerez, 1988; Toledo y Ordóñez, 1993; Challenger, 1998).

El bosque mesófilo de montaña, además, proporciona muchos servicios ambientales importantes. La captación de agua por este tipo de bosque, puede reducir el escurrimiento pluvial y aumentar la recarga de los mantos acuíferos, resultando en un aumento de la cantidad de agua disponible entre un 7 y un 158\% de la precipitación anual (Stadtmüller, 1987) y disminuir las inundaciones y sequías que dañan a los sistemas agropecuarios. El bosque de niebla igualmente contribuye de manera significativa al enriquecimiento $y$ desarrollo de los suelos debido a su baja tasa de descomposición (Challenger, 1998). También, los bosques saludables son muy importantes en la purificación del agua y para controlar la erosión de los suelos, el azolve de los ríos y el riesgo de inundaciones y deslaves, como las que recientemente han afectado varios estados del sureste de la República Mexicana (Myers, 1997). Finalmente, debido a su estructura compleja y a la alta biomasa por hectárea, existe mucho potencial en utilizar el bosque de niebla como parte de una estrategia nacional para recibir créditos en el mercado global del secuestro de carbono (De Jong et al., 2000; Challenger, 2001).

A pesar de lo importante de su biodiversidad y los servicios ambientales que presta a nivel mundial, el bosque de niebla está sumamente amenazado y presenta la tasa de deforestación más alta entre los bosques de tipo tropical (Doumenge et al., 1995; Aldrich et al., 2000). Se estima que en México, más del $50 \%$ de los bosques de niebla han desaparecido (Challenger, 1998). Históricamente, Veracruz ha sido el cuarto estado del país con mayor proporción de este tipo de ecosistema, pero gran parte de este y otros tipos de bosque han sido convertidos a otros usos de suelo (FloresVillela y Gerez, 1988; Dirzo y García, 1992; Williams-Linera, 1992) lo cual ayuda a explicar que Veracruz tenga el mayor número de especies en peligro de extinción (Flores-Villela y Gerez, 1988).

La importancia de conservar los bosques mesófilos de montaña en la cercanía de la capital del estado de Veracruz es apremiante; mientras que la zona montañosa de Veracruz ocupa un $11.6 \%$ de la superficie del estado y cuenta con el 26\% de la población total; de 1987 
a 2000, la zona urbana aumentó en un $440 \%$ a 13171 ha (Manson, datos no publicados).

En los últimos años, un número creciente de estudios han demostrado que el bosque de niebla y su biodiversidad son particularmente sensibles a los cambios causados por la fragmentación (WilliamsLinera, 1992; Kattan et al., 1994, Restrepo y Gómez, 1998). Además de la destrucción del hábitat que disminuye las poblaciones de flora y fauna, al ser fragmentado el ecosistema, aumenta el riesgo de extinción local de las especies típicas de plantas y animales de este bosque, ya que son expuestas a una disminución de humedad y a los efectos de borde (más luz, viento y temperatura). Los efectos negativos de la fragmentación se presentan espacialmente a diferentes escalas y principalmente son: una alteración del microclima dentro y afuera del remanente de bosque y el aislamiento de cada fragmento de los otros parches remanentes (Saunders et al., 1991).

Es necesario considerar que independientemente de que se conserve el área de un bosque, si éste es fragmentado, se incrementa el efecto de borde de cada parche, así como las condiciones no favorables bióticas y abióticas, lo que provoca una reducción del hábitat disponible para las especies nativas (e.g., Williams-Linera et al., 1995; Kattan et al., 1994; Restrepo y Gómez, 1998). Además, la fragmentación puede impedir el movimiento de los individuos entre remanentes de bosque e interrumpir un proceso crítico para la preservación de la diversidad genética (Bierregaard et al., 1992; Moilanen y Hanski, 1998). Los umbrales a los que puede llevarnos una fragmentación mayor y un efecto de borde adicional, debido al impacto humano, son aún desconocidos para la distribución y persistencia de especies de plantas y animales (Matlack, 1993; With y Crist, 1995).
Debido a la importancia del bosque de niebla para la conservación de la biodiversidad mexicana, a sus numerosos servicios ambientales, a su fragilidad y a la alta tasa de destrucción, es fundamental considerar lo siguiente: la severidad de muchos de los efectos negativos de la fragmentación del bosque de niebla, depende del tipo de vegetación que circunda a los fragmentos forestales, por lo que es apremiante conocer el grado y patrón de fragmentación del bosque y los tipos de uso del suelo a un nivel regional. De igual manera urgen estudios del estado actual de este bosque en Veracruz para así fundamentar la planeación del desarrollo regional que considere la conservación de este ecosistema.

\section{OBJETIVOS}

\section{Objetivo general}

Determinar el grado y el patrón de fragmentación del bosque de niebla en el centro del estado de Veracruz y poner esta información disponible, a quien la requiera para investigación, desarrollo y elaboración de planes para la conservación, restauración y manejo de este ecosistema.

\section{Objetivos particulares}

Los objetivos particulares son: 1) estimar el número de fragmentos remanentes de bosque, su tamaño y distancia de los asentamiento humanos más próximos y la relación perímetro-área como estimación de efecto de borde; 2) determinar los tipos de uso del suelo que rodea a los remanentes del bosque de niebla; y 3) contrastar las condiciones particulares de los fragmentos que se están utilizando para investigación con las condiciones generales de todos los remanentes para verificar la exactitud de las extrapolaciones que pudieran hacerse a nivel regional. 


\section{ÁREA DE ESTUDIO}

En la región central del estado de Veracruz se presenta un fuerte gradiente altitudinal; la elevación aumenta de 0 a $4300 \mathrm{msnm}$ en sólo $100 \mathrm{~km}$, desde la costa hasta la cima del Cofre de Perote. En esta porción del territorio, entre los 1200 y $2100 \mathrm{~m}$ de altitud, se localiza el bosque mesófilo de montaña (BMM) o bosque de niebla. El área de estudio se ubica al oeste de la ciudad de Xalapa y se extiende de 19 $30^{\prime} 01.03^{\prime \prime}$ a 19 $36^{\prime}$ '05.87" latitud norte y $96^{\circ}$ 54' 14.20" a 97- 02' 43.11" longitud oeste. Se seleccionó porque contiene a la mayoría de los últimos fragmentos de bosque de niebla en la región.
La zona localizada al este de Xalapa no se incluyó dado que tiene otro tipo de suelo (tepetates) y originalmente la vegetación fue encinar (Marchal y Palma, 1985). El espacio seleccionado incluye los municipios de Acajete, Banderilla, Coatepec, Rafael Lucio, San Andrés Tlalnehuayocan y Xalapa. En esta región, en cinco remanentes del bosque se han estudiado aspectos como estructura de la vegetación, microambiente, biodiversidad vegetal y de vertebrados, regeneración, fenología e interacciones bióticas. Los cinco sitios se localizan a una distancia media de $4 \mathrm{~km}$ entre ellos; algunas de sus características se presentan en la Tabla 1.

Tabla 1. Características de los cinco remanentes de bosque de niebla estudiados en el centro de Veracruz, México (Williams-Linera, 2002)

\begin{tabular}{|c|c|c|c|c|c|}
\hline \multicolumn{6}{|c|}{ FRAGMENTOS DE BOSQUE } \\
\hline Lugar & Parque & Xolostla & $\begin{array}{c}\text { Rancho } \\
\text { Viejo }\end{array}$ & Banderilla & $\begin{array}{l}\text { Mesa } \\
\text { Yerba }\end{array}$ \\
\hline Municipio & Xalapa & S. Andrés T. & S. Andrés T. & Banderilla & Acajete \\
\hline Altitud (m) & 1250 & 1450 & 1500 & 1470 & 1875 \\
\hline Latitud norte & $19^{\circ} 30^{\prime}$ & $19^{\circ} 32^{\prime}$ & $19^{\circ} 30^{\prime}$ & $19^{\circ} 35^{\prime}$ & $19^{\circ} 33^{\prime}$ \\
\hline Longitud oeste & $96^{\circ} 56^{\prime}$ & $96^{\circ} 58^{\prime}$ & $97^{\circ} 00^{\prime}$ & $96^{\circ} 56^{\prime}$ & $97^{\circ} 01^{\prime}$ \\
\hline Estado de protección & $\mathrm{R}$ & $\mathrm{P}$ & $P$ & $P$ & $P^{*}$ \\
\hline Área (ha) & 29.0 & 6.6 & 19.8 & 6.2 & 4.0 \\
\hline Poblado a menos de $1 \mathrm{~km}^{\star *}$ & sí & sí & no & sí & no \\
\hline Precipitación total anual (mm) & 1517 & 1650 & 1650 & 1451 & 1350 \\
\hline Temperatura media anual $\left({ }^{\circ} \mathrm{C}\right)$ & 18 & 16 & 14 & 16 & 12 \\
\hline \multicolumn{6}{|c|}{ CARACTERÍSTICAS DEL SUELO } \\
\hline $\mathrm{pH}$ & 3.8 & 4.2 & 3.9 & 4.7 & 3.7 \\
\hline Materia orgánica (\%) & 12 & 21.8 & 24.7 & 21.3 & 19.6 \\
\hline Compactación $\left(\mathrm{kg} / \mathrm{cm}^{2}\right)$ & 11.1 & 11.5 & 12.5 & 11.3 & 10.1 \\
\hline Densidad aparente $\left(\mathrm{g} / \mathrm{cm}^{3}\right)$ & 0.73 & 0.82 & 0.51 & 0.83 & 0.53 \\
\hline $\begin{array}{l}\mathrm{R} \text { reserva } \\
\mathrm{P} \text { propiedad privada }\end{array}$ & & $\begin{array}{ll}\mathrm{P}^{*} & \mathrm{~d} \\
* * & \mathrm{p}\end{array}$ & cona & $\begin{array}{l}\text { ervación } \\
00 \text { habitante }\end{array}$ & \\
\hline
\end{tabular}


En estos fragmentos de BMM, se identificó que las especies dominantes de árboles son Liquidambar styraciflua, Quercus xalapensis, Q. leiophylla, Q. germana y Clethra mexicana y árboles más pequeños como Turpinia insignis, Cinnamomum effusum, Carpinus caroliniana y Oreopanax xalapensis. Algunos arbustos presentes en varios sitios son Palicourea padifolia, Eugenia xalapensis, Miconia glaberrima y Ocotea psychotrioides (Williams-Linera, 2002). Una evidencia de la extinción de especies en la región es el hecho de que en estos fragmentos de bosque las especies de mamíferos pequeños que aún se encuentran representan sólo un $56 \%$ de las especies presentes en la región a principio del siglo pasado; ejemplos de algunas especies abundantes son Peromyscus furvus, $P$. aztecus, Microtus quasiater y Reithrodontomys magalotis (Manson, 2001).

La destrucción acelerada del bosque regional es un fenómeno reciente. A principios del siglo $X X$ la región situada en las cercanías de Xalapa presentaba grandes extensiones de bosque continuo. La parte central de Veracruz ha estado habitada desde tiempos prehispánicos por diferentes pueblos. Xalapa tuvo su origen alrededor de 1116 DC., pero durante los 400 años que siguieron, el poblado tuvo leve crecimiento físico y demográfico. En 1558 se encuestaron 1295 tributarios. En 1784 la localidad tenía una población de 7264 habitantes. A finales de la época colonial, la población alcanzaba los 13000 habitantes. En 1855, Xalapa fue declarada capital del Estado.

Este periodo se caracterizó por un desarrollo urbano muy lento y estable en el cual se consolidó la estructura de la ciudad. Entre el inicio del movimiento independiente e inicios del siglo pasado (XX) la población aumentó en sólo 7000 habitantes (González de Cossío, 1957). Desde principios del siglo XX y hasta 1980, la superficie urbana creció 19 veces y la población 10 veces (Marchal y
Palma, 1985). Los datos sobre crecimiento territorial y demográfico indican que la explosión de crecimiento urbano se disparó a partir de la década de 1960. Según Marchal y Palma (1985), el crecimiento se debió a flujos migratorios y clasificaron a Xalapa como un centro de atracción migratoria muy elevada.

El bosque mesófilo de montaña en la región central del estado de Veracruz se fue reduciendo gradual y paralelamente a la expansión del cultivo de café desde finales del siglo pasado hasta 1960 y posteriormente con la conversión a potreros para producción lechera (Marchal y Palma, 1985); el impacto ecológico fue hasta entonces relativamente leve. En contraste, a partir de los años 60's la destrucción del bosque fue muy acelerada debido a la extrema presión ejercida por el crecimiento explosivo, tanto demográfico como territorial de la ciudad de Xalapa y a sistemas recientes de ganadería lechera en superficies mayores (Marchal y Palma, 1985) (Fig. 1).

\section{METODOLOGÍA}

Se obtuvo un mapa de vegetación y uso del suelo a partir de la fotointerpretación y posterior digitalización de los contornos en un fotomosaico ortorectificado compuesto de treinta y tres fotografías aéreas de la región escala 1:20 000 (INEGI, 1993). Los tipos de cubierta vegetal se clasificaron en las siguientes categorías: bosque no perturbado, bosque perturbado (incluye cafetales con sombra), vegetación secundaria, pastizal, otros tipos y uso urbano. A partir de la cartografía topográfica digitalizada escala 1:50 000 (INEGI, 1995), hojas Xalapa (clave E14B27) y Perote (E14B26), se realizaron los análisis de pendientes (SLOPE) y orientación de las pendientes (ASPECT) del módulo Surface Analysis de IDRISI 4.1 (Eastman, 1992), posteriormente se exportaron a ARC/INFO 3.5 (ESRI, 1996). La mancha urbana de la 
ciudad de Xalapa se obtuvo de INEGI (INEGI, 1995). Los mapas se integraron en un sistema de información geográfica (SIG) PC ARC/INFO 3.5. Otra información integrada al SIG fueron los rasgos topográficos y asentamientos humanos. Se generaron varios mapas que se verificaron mediante visitas al campo. Los mapas que se incluyen en este estudio son: uso del suelo, poblaciones, porcentaje de pendiente $\left(100 \%=90^{\circ}\right)$ y orientación de la pendiente ( $\mathrm{N}$ va de 315 a $45^{\circ}$, $\mathrm{E}$ va de 45 a $135^{\circ}$, S va de 135 a $225^{\circ}$, y O va de 225 a 315).

Para determinar las distancias de los fragmentos de bosque no perturbado a los asentamientos humanos, los datos se analizaron utilizando el comando NEAR de PC ARC/INFO. Este comando estimó la distancia entre cada localidad al arco más cercano de un polígono (fragmento) de bosque no perturbado.

En una segunda etapa, los mapas vectoriales generados en el programa PC ARC/INFO se importaron a GRASS 4.0 en formato raster (imagen) con una resolución de pixel de 20 × $20 \mathrm{~m}$.
Utilizando estos mapas, se estimó el área y perímetro de cada fragmento de bosque no perturbado y se estimaron dos proporciones. La primera proporción fue perímetro-área de cada fragmento. Dado que el círculo es la figura geométrica que minimiza esta proporción, se utilizó la fórmula del círculo para calcular el perímetro esperado a partir del área de cada fragmento si fuera circular. Se compararon la proporción perímetro real y esperado para cada fragmento. Mientras más parecida era la forma del fragmento a un círculo del mismo tamaño (100\% de concordancia), el fragmento tendría menos perímetro y por lo tanto menos efecto de borde (modificado de Forman, 1997).

Para analizar los patrones del uso de suelo adyacente a cada fragmento, se consideraron dos zonas de amortiguamiento, a 100 y $1000 \mathrm{~m}$, a partir del borde de cada fragmento de bosque no perturbado. El mapa con esas dos zonas se sobrepuso al mapa de vegetación y uso del suelo. A partir de lo anterior, se calculó el porcentaje de área que cada tipo de uso del suelo ocupó dentro de cada zona.

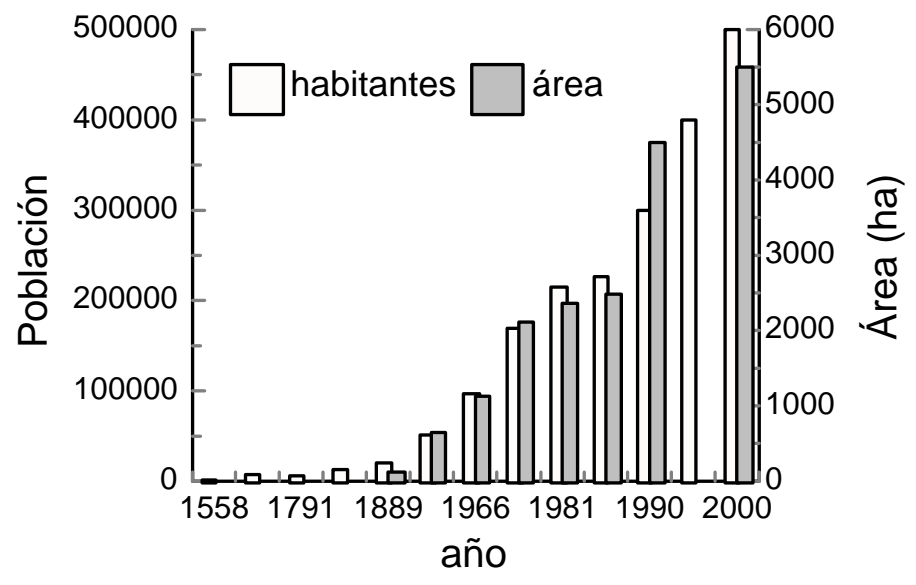

Figura 1. Crecimiento poblacional y territorial de la ciudad de Xalapa, Ver., Méx., durante los últimos 400 años (Fuentes: González de Cossío, 1957; Marchal y Palma, 1985; censos 1990, 1995, 2000). 


\section{RESULTADOS}

En la región oeste de Xalapa, Veracruz, quedan solamente 19 fragmentos de bosque de niebla no perturbado según el análisis del SIG. La mayor parte de bosque no perturbado se detectó en el cuadrante suroeste de la región de estudio (Fig. 2). Los fragmentos de bosque no perturbado cubren únicamente el $10 \%$ del área estudiada y el único fragmento de tamaño grande tenía 902 ha en 1993 (Tabla 2).

El uso de suelo dominante en la región es el pastizal $(37 \%$ de la superficie), seguido por zonas urbanas (18\%), vegetación secundaria (17\%) y bosque perturbado (17\%). Otras coberturas representaron el $2 \%$ de la superficie (Tabla 2). El número de polígonos contados así como el tamaño medio, mínimo y máximo según los tipos de uso del suelo se presentan en la Tabla 2.

Las categorías de pendiente más comunes en la región fueron 0 al 5\% (37\% del área total) y 5 al 10\% (27\%). La orientación principal de las pendientes a nivel regional es sur $(33 \%)$ y este $(30 \%$, Fig. 3). Existe muy poca superficie plana en la región al oeste de Xalapa (3.2\%). Sin embargo, esta pendiente plana es relativamente más común para usos urbanos $(76 \%$ de superficie dedicada a uso urbano) y pastizales (39\% de área ocupada por pastizales) mientras que el $39 \%$ del área de bosque no perturbado se encuentra en zonas con pendientes muy pronunciadas. La pendiente y la orientación de la misma en cada categoría de uso del suelo se presentan en la Tabla 2. Los fragmentos de bosque no perturbado tienen una pendiente más fuerte y una orientación de pendientes más hacia el norte de la que se observó a nivel regional (Fig. 3).

Existe una relación negativa entre el tamaño de los fragmentos de bosque no perturbado y el tamaño de los asentamientos humanos. Además, mientras mayor es el número de habitantes, más grande es la distancia que separa los asentamientos humanos y el bosque más cercano (Fig. 4). Los fragmentos de bosque no perturbado y de bosque perturbado mostraron una tendencia similar con respecto a estas relaciones (Fig. 4).

Los remanentes de bosque más pequeños tuvieron una forma más parecida a la del círculo que los fragmentos más grandes y se encontró una relación negativa significativa entre el área de los fragmentos y el perímetro correspondiente a un círculo de la misma área (Fig. 5). La regresión lineal es significativa e indica que los fragmentos de mayor tamaño tienen una forma menos parecida a la del círculo y por lo tanto más borde. En promedio, la forma de los fragmentos fue diferente a la del círculo, por lo que su perímetro $\left(P_{1}\right)$ resultó casi tres veces más largo de lo que correspondería al perímetro de un círculo $\left(P_{2}\right)$ con la misma superficie $\left(P_{1}: P_{2}=2.7\right)$. Debido a que el efecto de borde se extiende alrededor de $15 \mathrm{~m}$ hacia el interior del fragmento (Williams-Linera, 1993), en general, hay una disminución de $15.3 \%$ del área real del bosque no perturbado, lo cual reduciría la cobertura regional en un $8.5 \%$. Sin embargo, para los fragmentos más pequeños (p.e.: una hectárea) la disminución real alcanza cerca de la mitad de la superficie de bosque $(54 \%)$ debido al efecto de borde.

Los fragmentos de bosque están rodeados por usos del suelo en una tendencia semejante a la proporción de usos del suelo que se presentan en la región (Tabla 2, Fig. 6a). Se observó la misma tendencia para todos los remanentes en el intervalo de tamaños existentes. Los potreros cubren un promedio del $59 \%$ del área que rodea a los fragmentos, mientras que el bosque perturbado y la vegetación secundaria ocupan un área similar alrededor de los fragmentos (ca. 19\%), aunque el uso urbano cubre sólo $1 \%$. 


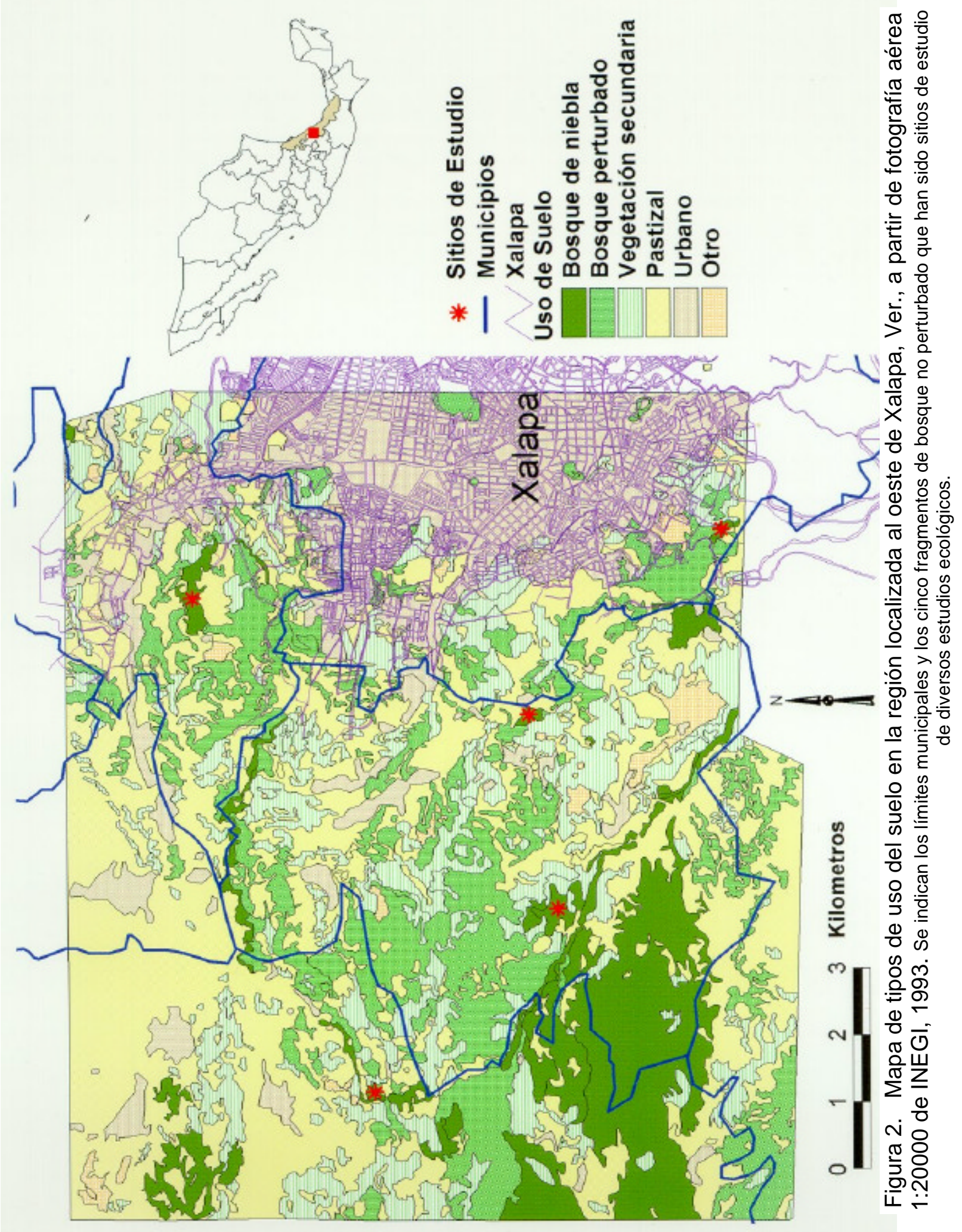


Tabla 2. Resultados de sistema de información geográfica para la región oeste de Xalapa, Veracruz

\begin{tabular}{|c|c|c|c|c|c|c|}
\hline $\begin{array}{l}\text { VARIABLES Y } \\
\text { CATEGORÍA }\end{array}$ & $\begin{array}{l}\text { BOSQUE NO } \\
\text { PERTURBADO }\end{array}$ & $\begin{array}{c}\text { BOSQUE } \\
\text { PERTURBADO }\end{array}$ & $\begin{array}{l}\text { VEGETACIÓN } \\
\text { SECUNDARIA }\end{array}$ & PASTIZALES & TROS & $\begin{array}{c}\text { USO } \\
\text { URBANO }\end{array}$ \\
\hline \multicolumn{7}{|l|}{ SUPERFICIE } \\
\hline $\begin{array}{l}\text { No. de } \\
\text { polígonos }\end{array}$ & 19 & 104 & 149 & 177 & 32 & 42 \\
\hline Área total & 1287 & 2149 & 2189 & 4749 & 204 & 2266 \\
\hline Porcentaje & 0.10 & 0.17 & 0.17 & 0.37 & 0.02 & 0.18 \\
\hline Media & 67.7 & 20.7 & 14.7 & 26.8 & 6.4 & 53.9 \\
\hline Mínima & 0.93 & 0.03 & 0.13 & 0.01 & 0.33 & 0.59 \\
\hline Máxima & 902 & 732 & 157 & 1916 & 66 & 1901 \\
\hline \multicolumn{7}{|c|}{ PENDIENTE (\%) } \\
\hline $0-5$ & 0.13 & 0.21 & 0.23 & 0.39 & 0.39 & 0.76 \\
\hline $5-10$ & 0.21 & 0.28 & 0.28 & 0.31 & 0.32 & 0.18 \\
\hline $10-15$ & 0.22 & 0.22 & 0.22 & 0.16 & 0.17 & 0.05 \\
\hline $15-30$ & 0.39 & 0.27 & 0.25 & 0.14 & 0.12 & 0.02 \\
\hline$>30$ & 0.05 & 0.03 & 0.02 & 0.00 & 0.00 & 0.00 \\
\hline \multicolumn{7}{|c|}{ ORIENTACIÓN DE LA PENDIENTE } \\
\hline Norte & 0.31 & 0.23 & 0.22 & 0.20 & 0.15 & 0.16 \\
\hline Este & 0.29 & 0.26 & 0.28 & 0.36 & 0.29 & 0.26 \\
\hline Sur & 0.31 & 0.35 & 0.33 & 0.32 & 0.31 & 0.34 \\
\hline Oeste & 0.04 & 0.10 & 0.09 & 0.05 & 0.11 & 0.13 \\
\hline Plano & 0.04 & 0.07 & 0.08 & 0.07 & 0.13 & 0.11 \\
\hline \multicolumn{7}{|l|}{ POBLACIÓN } \\
\hline $\begin{array}{l}\text { No. } \\
\text { habitantes }\end{array}$ & \multicolumn{6}{|c|}{ Distancia de la población al fragmento de bosque más cercano $(\mathrm{km})$} \\
\hline $6-100$ & 0.73 & 0.48 & & & & \\
\hline $101-1000$ & 0.73 & 0.33 & & & & \\
\hline $1001-10000$ & 1.55 & 0.50 & & & & \\
\hline$>10000$ & 2.51 & 1.19 & & & & \\
\hline $\begin{array}{l}\text { No. } \\
\text { habitantes }\end{array}$ & \multicolumn{6}{|c|}{ Superficie del fragmento de bosque más cercano a la población (ha) } \\
\hline $6-100$ & 204.88 & 29.08 & & & & \\
\hline $101-1000$ & 127.96 & 9.25 & & & & \\
\hline $1001-10000$ & 13.00 & 7.72 & & & & \\
\hline$>10000$ & 23.89 & 7.38 & & & & \\
\hline
\end{tabular}


a)

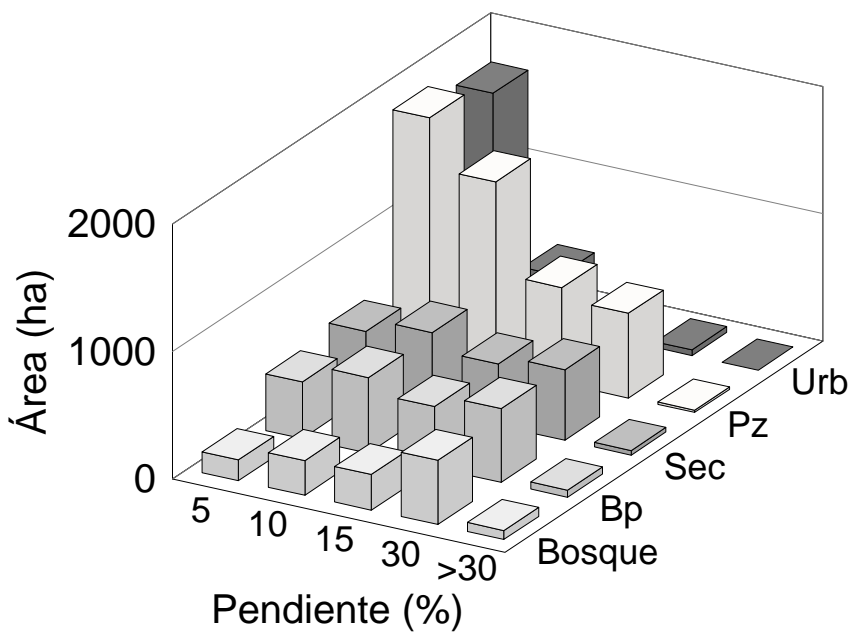

b)

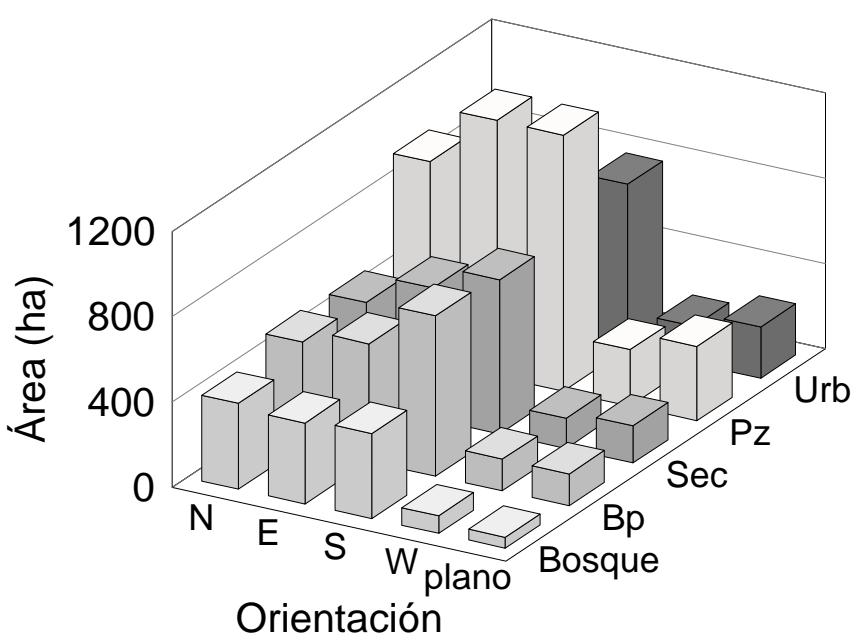

Bosque: fragmentos de bosque no perturbado

$B p$ : bosque perturbado

Sec: vegetación secundaria

Pz: pastizal Urb: usos urbanos

Figura 3. Área ocupada por los diferentes tipos de uso de suelo en la región al oeste de Xalapa, de acuerdo con a) la pendiente y b) la orientación de la pendiente.

Al enfocarse en una escala espacial mayor, 1000 y $100 \mathrm{~m}$ alrededor de cada fragmento, se encontró la misma tendencia, aunque en la zona de amortiguamiento de $100 \mathrm{~m}$, hay un aumento en el porcentaje de pastizales y vegetación secundaria rodeando los fragmentos así como una disminución en el uso del suelo de bosque perturbado (Fig. 6b, c).

A una escala de paisaje, los cinco fragmentos seleccionados para investigación se encuentran rodeados por una proporción de usos del suelos semejante a la media de los 19 fragmentos de bosque no perturbado (Fig. 6a). Al cambiar a la escala espacial de enfoque de $1000 \mathrm{~m}$ alrededor de los fragmentos, hubo una tendencia a que los sitios de estudio estuvieran rodeados de un porcentaje de uso urbano mayor que la de todos los fragmentos (Fig. 6b). A $100 \mathrm{~m}$ del borde, la tendencia fue un mayor porcentaje de bosque perturbado que rodea los sitios de estudio que el porcentaje promedio rodeando a todos los fragmentos (Fig. 6c). 
a)

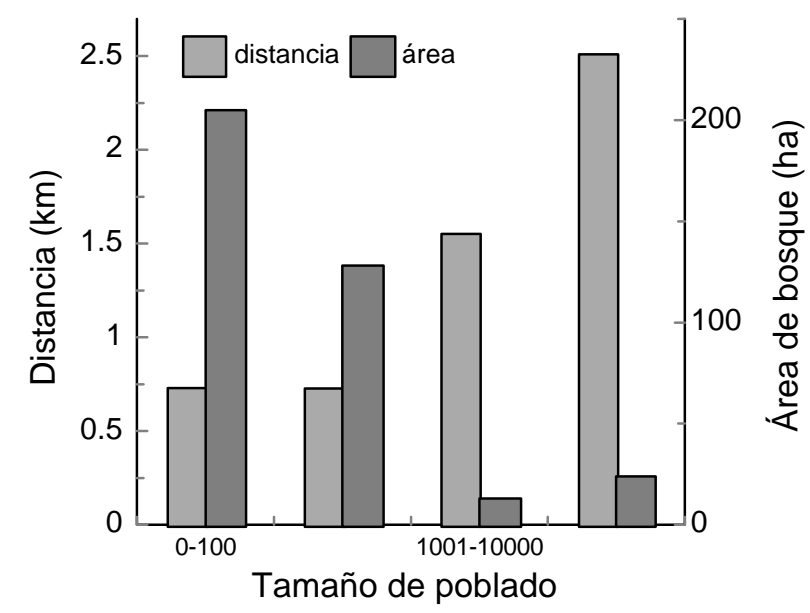

b)

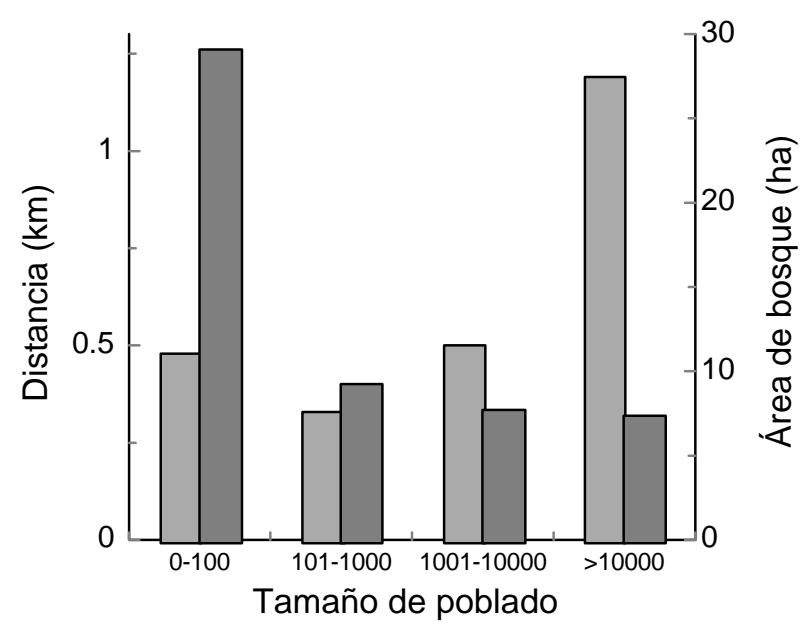

Figura 4. Distancia y área de fragmentos de bosque a asentamientos humanos con diferente número de pobladores en la región al oeste de Xalapa, Veracruz. a) Fragmentos de bosque no perturbado y b) Fragmentos de bosque perturbado.

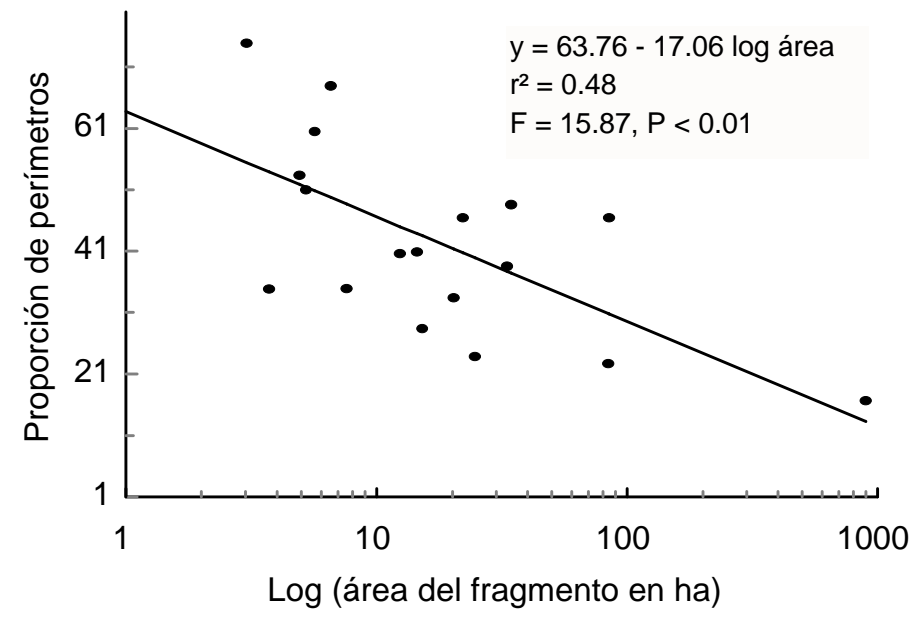

Figura 5. Área de los fragmentos de bosque no perturbado en la región al oeste de Xalapa, Veracruz, y la proporción entre el perímetro del fragmento y el perímetro de un círculo con la misma área $\left(\mathrm{P}_{1} / \mathrm{P}_{2}\right)$. 
a)

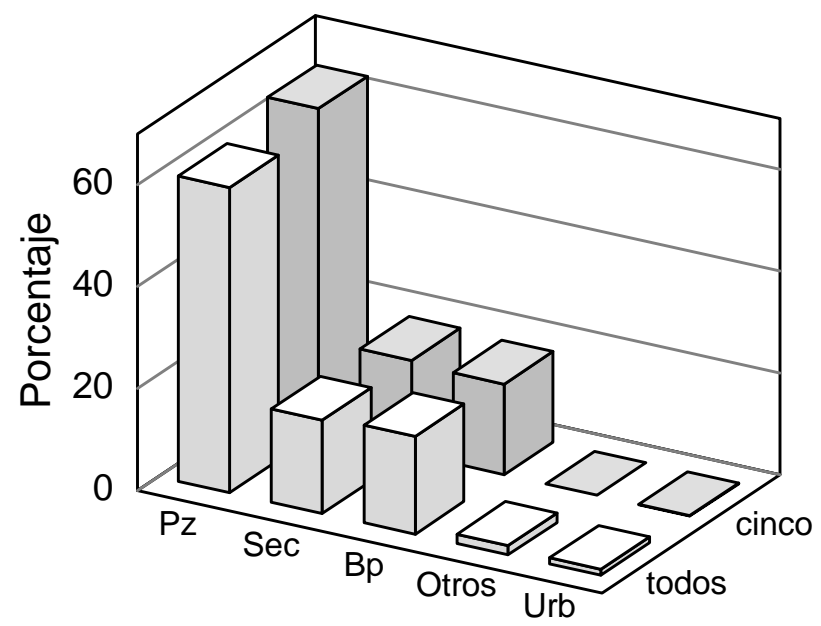

b)

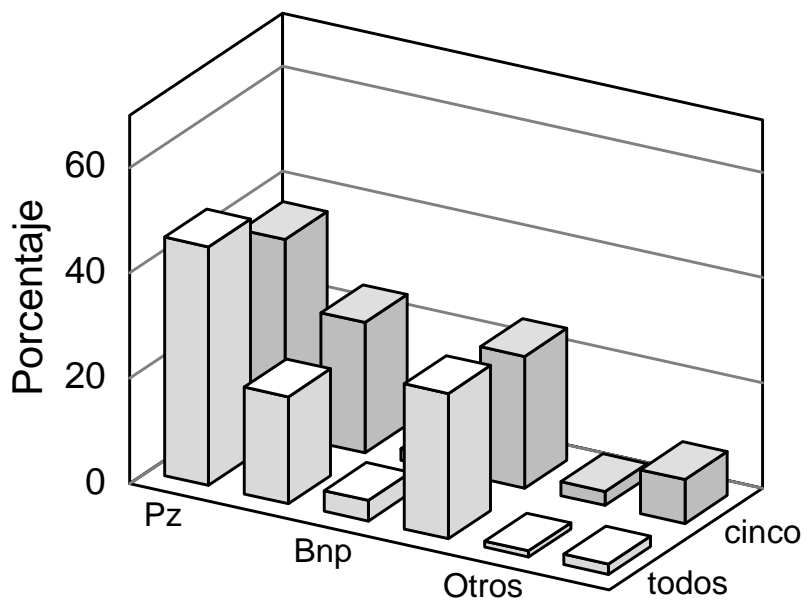

Pz: pastizal

Sec: vegetación secundaria

Bnp: bosque de niebla no perturbado

$B p$ : bosque perturbado Urb: usos urbanos todos: promedio de los 19 fragmentos de bosque encontrados en la región

cinco: promedio de los cinco fragmentos utilizados como sitios de estudio del bosque de niebla

c)

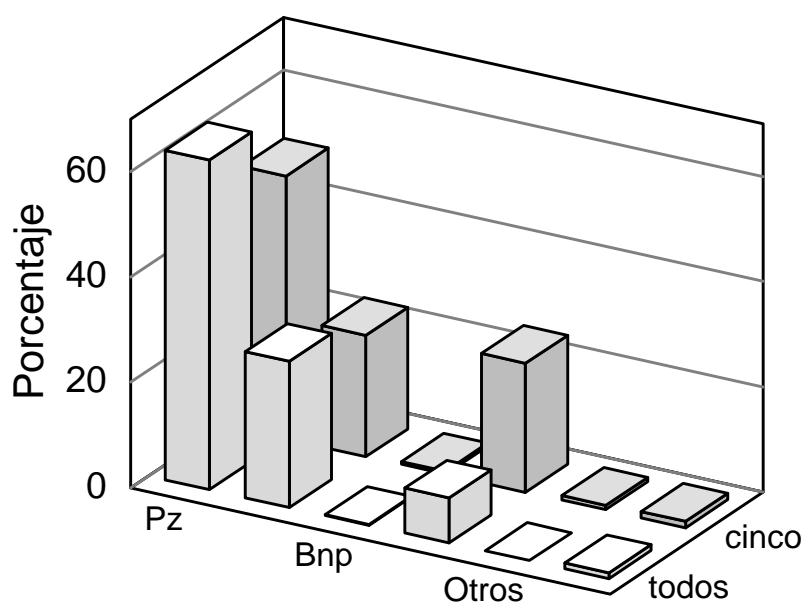

Figura 6. Porcentaje de usos del suelo en la región al oeste de Xalapa, Veracruz, a) al borde de los fragmentos de bosque de niebla no perturbado, b) en una zona de amortiguamiento de $1000 \mathrm{~m}$ alrededor de los fragmentos y c) en una zona de $100 \mathrm{~m}$ alrededor de los fragmentos. 


\section{DISCUSIÓN}

Históricamente, la región al oeste de Xalapa ha estado cubierta por bosque mesófilo de montaña, según consta en crónicas de viajeros y colectores botánicos que visitaron la región en los últimos siglos (González de Cossio, 1957). Los resultados de este SIG indican una pérdida del $90 \%$ de este tipo de bosque en la región de estudio, lo cual implica que la destrucción de bosque de niebla ha sido más alta a nivel nacional (Challenger, 1998) o mundial (Doumenge et al., 1995). La pérdida de bosques debido al crecimiento urbano es un fenómeno documentado a nivel global. Por ejemplo, en Estados Unidos las áreas urbanas han engullido 13 millones de acres de tierra entre 1970 y 1980 y otros 9 millones de acres en los 80's; estas tierras estaban dedicadas a usos agrícolas y ecosistemas frágiles forestales, desierto y humedales (Parlange, 1998).

En Puerto Rico, el crecimiento urbano también está ocurriendo con tasas altas sobre tierras agrícolas (López et al., 2001) y está, actualmente, entrando en áreas con bosque (Thomlinson et al., 1996). En la región de Xalapa, el agravante es que esta destrucción ha ocurrido exponencialmente en los últimos 50 años, ya que la reducción histórica del bosque fue gradual en los últimos 500 años (Fig. 1) y que esta expansión está aconteciendo más hacia el oeste de la ciudad donde existen suelos más fértiles y pendientes más fuertes y por lo tanto el riesgo de erosión es más alto.

El fragmento de bosque no perturbado de mayor tamaño tenía, en la fotografía aérea de 1993, 902 ha de superficie, sin embargo, diariamente se extrae madera de arrastre de esta área y por lo tanto actualmente su superficie debe ser menor y debe estar perturbado. Los fragmentos de bosque se encuentran relativamente alejados de zonas urbanas pero el crecimiento de la ciudad de Xalapa hacia el oeste y la apertura de carreteras pone en verdadero peligro de desapa- rición a este último remanente del bosque original. En otros bosques se ha documentado que la apertura de caminos son el agente causal de la desaparición del bosque debido a la fragmentación y al acceso fácil para la deforestación y colonización (p.e. Costa Rica: Sader y Joyce, 1988; Perú: Young, 1994).

El efecto de borde que se estimó es el originado únicamente por cambio en condiciones microambientales y de vegetación, sin embargo, el impacto humano sobre fragmentos se presenta en mayor escala hacia el interior del bosque. Matlack (1993) estimó que el impacto humano puede ocurrir hasta $70 \mathrm{~m}$ en el interior del bosque $y$, que este efecto se presenta aún más adentro si hay acceso para vehículos; además, los efectos humanos son considerablemente más dañinos que los efectos naturales de borde y al contrario que factores como luz y temperatura no disminuyen con la distancia del borde, por lo cual el área real de bosque es mucho menor de la que se ha estimado.

Los remanentes se encuentran en pendientes que, en promedio, son más abruptas que las del paisaje en general, lo que confirma las observaciones anecdóticas de varios autores que han considerado que los bosques se encuentran en lugares poco accesibles y menos útiles para otros tipos de uso de suelo. En Costa Rica, una situación similar se ha documentado y se encontró que el bosque primero se removió completamente de las pendientes suaves (0-5\%) pero que actualmente aún en las pendientes más abruptas (>60\%) se cuenta con menos de una tercera parte del área total del bosque original (Sader y Joyce, 1988). En este contexto (la región es, en general, muy montañosa) las zonas de pendientes fuertes deberían ayudar a detener la expansión urbana y el impacto humano sobre el bosque.

Los fragmentos de bosque no perturbado se encuentran aislados y sus formas son alargadas y complejas lo cual 
aumenta el efecto de borde. Los fragmentos pequeños tienen formas más circulares y por lo tanto más efecto de borde natural y humano, lo cual reduce el área real de bosque. Además, una proporción alta de la cobertura del suelo es pastizal lo cual aísla aún más a muchos organismos que no son capaces de cruzar esta matriz de vegetación tan diferente al bosque. El cambio en el patrón de uso de suelo de 100 a $1000 \mathrm{~m}$ del borde de los fragmentos de bosque muestra la complejidad que enfrentan las especies que intenten cruzar la matriz de pastizales.

Es importante señalar el hecho de que existen todavía muchos fragmentos con bosques perturbados y vegetación secundaria en la región. Estas coberturas se podrían utilizar como corredores para ayudar a conectar los fragmentos de bosque no perturbado. Sin embargo, sólo con programas de reforestación bien ejecutados y a escalas más grandes de las que se han realizado en el pasado se podría reducir significativamente el aislamiento de estos remanentes de bosque. Los sitios de estudio de este trabajo son similares en sus características a los fragmentos de bosque no perturbado que se encuentran en la región, por lo que los resultados obtenidos se pueden generalizar a nivel de la región.

En el futuro se requiere más investigación para: 1) vincular los estudios de fragmentación a nivel paisaje con cambios en la fauna y flora dentro de los fragmentos de bosque mesófilo de montaña en la región, 2) determinar la tasa y los patrones de cambio en uso de suelo dentro de la región para poder predecir impactos potenciales sobre los manchones de bosque remanente y 3) cuantificar los servicios ambientales proporcionados por el bosque para poder evaluar el impacto económico real que causará la pérdida de estos ecosistemas sobre la calidad de vida. El uso de modelos, para mostrar a la ciudadanía los impactos potenciales de sus acciones en el futuro, podría realmente ayudar a mitigar las presiones de la población sobre los recursos y a entender las interacciones que existen entre la ecología, el clima y los sistemas sociales y económicos (Parlange, 1998). Este tipo de estudios se están llevando a cabo como estudios ecológicos a largo plazo considerando a la "ciudad como ecosistema" en Phoenix y Baltimore, Estados Unidos. Dadas las condiciones ecológicas y sociales de Xalapa, sería posible proponerla para que formará parte de este tipo de estudios.

\section{CONCLUSIONES}

El bosque mesófilo de montaña del centro de Veracruz es un ecosistema que está en peligro de desaparecer. En 1993 sólo se contaba con el $10 \%$ del bosque original de la región, cifra que seguramente ha disminuido en los últimos años. Una amplia verificación de campo y los datos del sistema de información geográfica indican que la situación en la región es crítica: sólo quedan 19 fragmentos de bosque de niebla relativamente no perturbado. Sin embargo, aún se encuentran muchos fragmentos de bosque perturbado (104). La conservación de los remanentes del bosque, así como la rehabilitación y restauración ecológica de los fragmentos perturbados es necesaria como parte de un plan de desarrollo regional que considere la conservación de la biodiversidad y de los servicios ambientales del bosque en la región. Parte de la estrategia debe tener como misión detener la expansión urbana hacia el oeste de Xalapa y dirigirla hacia el este, donde las pendientes son menos pronunciadas y los suelos menos frágiles, aumentar la conectividad entre los fragmentos existentes y tener en cuenta que la divulgación de estas actividades es una tarea importante y es un punto crucial de cualquier estrategia. 


\section{RECONOCIMIENTOS}

Agradecemos los valiosos comentarios de tres revisores anónimos. El presente estudio se llevó a cabo con el apoyo de la Comunidad Europea bajo el programa INCO-DC (Framework 4) como parte del proyecto SUCRE (ERBIC18CT97-0146) y del Departamento de Ecología Vegetal (IE 902-16) del Instituto de Ecología, A. C.

\section{REFERENCIAS}

Aldrich, M.; P. Bubb; S. Hostettler y H. van de Wiel. 2000. Bosques nublados tropicales montanos. Tiempo para la acción. WWF International/IUCN The World Conservation Union. Cambridge. Inglaterra. 28 p.

Bierregaard, R.O.; T.E. Lovejoy; V. Kapos; A.A dos Santos y R.W. Hutchings. 1992. The biological dynamics of tropical forest fragments. Bioscience 42:859-866.

Challenger, A. 1998. Utilización y conservación de los ecosistemas terrestres de México. Pasado, presente y futuro. Comisión Nacional para el Conocimiento y uso de la Biodiversidad. UNAM Agrupación Sierra Madre, S. C. México, D.F. 847 p.

Challenger, A. 2001. ¿Qué es el bosque mesófilo de montaña? pp. 20-26. In: R. H. Manson y G. Williams-Linera, eds. Memorias del taller sobre conservación y uso sustentable del bosque mesófilo de montaña en el centro de Veracruz. Subsecretaría del Medio Ambiente. Instituto de Ecología, A.C. Xalapa, Ver. 79 p.

Dirzo, R. y M.C. García. 1992. Rates of deforestation in Los Tuxlas, a Neotropical area in southeast Mexico. Conservation Biology 6: 84-89.
De Jong, B.H.J., R. Tipper y G. MontoyaGómez. 2000. An economic analysis of the potential for carbon sequestration by forests: evidence from southern Mexico. Ecological Economics 33:313-320.

Doumenge, C.; D. Gilmour; M.P. Ruíz y J. Blockhus. 1995. Tropical montane cloud forests: conservation status and management issues. pp. 24-37. In: L. S. Hamilton, J.O Juvik y F.N., Scatena, eds. Tropical Montane Cloud Forests. Springer-Verlag, Nueva York. 407 p.

Eastman, J.R. 1992. IDRISI 4.1. Source Code, IDRISI Production,1987-1992. Clark University.

ESRI. 1996. Environmental Systems Research Institute, Inc. PC ARC/INFO 3.5 .

Fa, J.E. y L.M. Morales.1993. Patterns of mammalian diversity in Mexico. pp. 319-361. In: T.P. Ramamoorthy; R. Bye; A. Lot y J. Fa, eds. Biological diversity of Mexico: origins and distribution. Oxford University Press. Nueva York. 812 p.

Flores-Villela, O. y P. Gerez. 1988. Conservación en México: Síntesis sobre vertebrados terrestres, vegetación y uso del suelo. INIREB y Conservation International. México, D.F. 302 p.

Forman, R.T.T. 1997. Land mosaics. The ecology of landscapes and regions. Cambridge University Press. Cambridge. Inglaterra p. 142.

González de Cossío, F. 1957. Xalapa. Breve reseña histórica. Talleres Gráficos de la Nación. México, D.F. 488 p.

Kattan, G.H.; H. Álvarez L. y M. Giraldo. 1994. Forest fragmentation and bird extinctions: San Antonio eighty years later. Conservation Biology 8:138-146. 
López, T.M.; T.M. Aide y J.R. Thomlinson. 2001. Urban expansion and the loss of prime agricultural lands in Puerto Rico. Ambio 30:49-54.

Manson, R.H. 2001. El bosque de niebla también consiste de animales peludos. Jarocho Verde 13/14:10-13.

Marchal, J.Y. y R. Palma. 1985. Análisis gráfico de un espacio regional: Veracruz. INIREB-ORSTOM. Xalapa, Ver. 220 p.

Matlack, G.R. 1993. Sociological edge effects: spatial distribution of human impact in suburban forest fragments. Environmental Management 17:829835.

Moilanen, A. e I. Hanski. 1998. Metapopulation dynamics: effects of habitat quality and landscape structure. Ecology 79:2503-2515.

Myers, N. 1997. The world's forests and their ecosystem services. pp. 215235. In: G.C. Daily, ed. Nature's services: societal dependence on natural ecosystems. Island Press. Washington, D.C. 416 p.

Parlange, M. 1998. The city as ecosystem. Bioscience 48:581-585.

Rzedowski, J. 1993. Diversity and origins of the fanerogamic flora of Mexico. pp. 139-144. In: T.P. Ramamoorthy, R. Bye, A. Lot y J. Fa, eds. Biological Diversity of Mexico: Origins and distribution. Oxford University Press. Nueva York. 812 p.

Rzedowski, J. 1996. Análisis preliminar de la flora vascular de los bosques mesófilos de montaña de México. Acta Botánica Mexicana 35:25-44.

Restrepo, C. y N. Gómez. 1998. Responses of understory birds to anthropogenic edges in a Neotropical montane forest. Ecological Applications 8:170-183.
Sader, S.A. y A.T. Joyce. 1988. Deforestation rates and trends in Costa Rica, 1940 to 1983 . Biotropica 20:11-19.

Saunders, D.A.; R.J. Hobbs y C.R. Margules. 1991. Biological consequences of ecosystem fragmentation: a review. Conservation Biology 5:1832.

Stadmüller, T. 1987. Los Bosques Nublados en el Trópico Húmedo. UNU, CATIE. Turrialba, Costa Rica. 85 p.

Thomlinson, J.R.; M.I. Serrano; T.M. López; T.M. Aide y J.K. Zimmerman. 1996. Land-use dynamics in a postagricultural Puerto Rican landscape (1936-1988). Biotropica 28:525-536.

Toledo, V.M. y M.J. Ordóñez. 1993. The biodiversity scenario of Mexico: a review of terrestrial habitats. pp. 757-777. In: T.P. Ramamoorthy, R. Bye, A. Lot y J. Fa, eds. Biological Diversity of Mexico: Origins and distribution. Oxford University Press. Nueva York. 812 p.

Williams-Linera, G. 1992. Ecología del paisaje y el bosque mesófilo de montaña en el centro de Veracruz. Ciencia y Desarrollo XVIII (105):132138.

Williams-Linera, G. 1993. Vegetación de bordes de un bosque nublado en el Parque Ecológico Clavijero, Xalapa, Veracruz, México. Revista de Biología Tropical 41:443-453.

Williams-Linera, G. 2002. Tree species richness complementarity, disturbance and fragmentation in a Mexican tropical montane cloud forest. Biodiversity and Conservation. En prensa.

Williams-Linera, G.; V. Sosa, y T. Platas. 1995. The fate of epiphytic orchids after fragmentation of a Mexican cloud forest. Selbyana 16:36-40. 
With, K.A. y T.O. Crist. 1995. Critical thresholds in species responses to landscape structure. Ecology 76: 2446-2459.
Young, K.R. 1994. Roads and the environmental degradation of tropical montane forests. Conservation Biology 8:972-976.»

1 Departamento de Ecología Vegetal, Instituto de Ecología, A. C., Apartado Postal 63. Xalapa 91000 Veracruz. México. c.e.: lupew@ecologia.edu.mx.

2 Sistema de Información Geográfica, Pladeyra, S. C. Allende 124 D-1. Xalapa 91000 Veracruz. México.

Manuscrito recibido el 7 de septiembre de 2001

Aceptado el 16 de noviembre de 2001

Este documento se debe citar como:

Williams L., G; R.H. Manson y E. Isunza V. 2002. La fragmentación del bosque mesófilo de montaña y patrones de uso del suelo en la región oeste de Xalapa, Veracruz, México. Madera y Bosques 8(1):73-89. 